One of the more unwieldy concepts with which social scientists struggle is that of power. How can one compare the power of a policeman to direct traffic with the power of a professor to get his students to do a homework assignment, or with the power of a father to get his son to wash behind his ears? The persons are so different, the behaviors so diverse, that they seem to preclude comparison. Is there a "common denominator" for these dissimilar situations? Here one approach to this problem is presented, using the common denominator of motivation.

\title{
AN EVENT-STRUCTURE APPROACH TO SOCIAL POWER AND TO THE PROBLEM OF POWER COMPARABILITY
}

\section{by Arnold S. Tannenbaum}

The University of Michigan 1

$\mathbf{A}$ SERIOUs problem confronting psychologists interested in the concept of power is that of comparability, the problem implicit in trying to compare the powers of individuals over others in relation to widely different acts. Dahl refers to differences in the types of behavior controlled as differences in scope, and states the problem as follows:

"In whatever fashion one chooses to define the relevant properties of the actors whose power he wishes to compare, strictly speaking one must compare them with respect to the responses they are capable of evoking. Ideally, it would be desirable to have a single measure combining differences in scope, number of comparable respondents controlled, and change in probabilities [of behavior]. But there seems to exist no intuitively satisfying method for doing so" (Dahl, 1957, p. 206).

Agreeing essentially with Dahl, Cartwright argues:

"In comparing the power of any two agents, it is clear that no single, unidimensional scale of magnitude will be adequate ... . Much further work will be required before an entirely satisfactory solution to this problem has been achieved" (Cartwright, 1959b, p. 210).

1 This paper was developed under a grant from the Carnegie Corporation of New York to the Survey Research Center, Institute for Social Research, The University of Michigan, for the study of control in social organizations. I am indebted to John R. P. French, Jr., F. H. Allport, D. Cartwright, Alvin Zander, J. Gyr, Clagett Smith, and S. S. West for useful questions and valuable suggestions. The author alone, however, is responsible for the interpretations presented in this paper.
Yet a simple fact suggests that a solution, in principle, may be possible. Behavior involves "choices," choices between qualitatively diverse alternatives. We make choices when we eat; we make choices when we speak, write, or read; we choose a residence, a job, a spouse. Each of these choices involves comparing alternatives which seem, on the face of it, incomparable. A high school graduate chooses among several jobs; one of which offers the advantage of better pay, another, more interesting work. How can money be seen as comparable in any way to interesting work? A man chooses a mate from among several eligible women. One is more attractive physically; a second has a more pleasing personality; a third is more intelligent. How can the prospective spouse compare physical beauty with the more "ethereal" qualities of personality, or with intelligence?

It is clear, however, that man does make these comparicons-whatever the dynamics of this process may be. This is an ostensible fact of human psychology. The explanation of this fact is less apparent. But the fact that such comparisons are natural to human beings suggests the existence of a "mechanism" within the organism which provides for the equilibration or standardization of qualitatively diverse stimuli, situations, and circumstances. It is this "mechanism," we propose, which provides the basis for a solution to the problem of power comparability and suggests an approach to a more adequate definition of power.

Social power has been variously defined, 
and "lifferent terms (e.g., control, influence, authority) are sometimes used synonymously with it. We accept as a starting point the conception suggested by Dahl (1957), who defines the power of $A$ over $a$ in terms of the probability that $a$ will do $x$ in response to $A$ 's request minus the probability that $a$ would do $x$ in the absence of $A$ 's request. We shall outline below an event-structure approach to power which represents a modification of Dahl's basic notion. Simply put, our approach takes into account two aspects of the controlled behavior in addition to Dahl's probabilities: (1) $a$ 's motivational energies involved in the controlled behavior $(x)$, and (2) a's motivational energies relative to other acts which $a$ cannot perform as a result of his acceding to $A$ 's request. Our notion of power thus takes into account not only what $A$ "makes" $a$ do, but what he "prevents" $a$ from doing. We consider in this way the total "importance" or "significance" to $a$ of performing the act in question. We propose that this factor of "importance," reflecting motivational energies within $a$, represents a step in the direction of a "common denominator" for comparing control over different acts under widely differing circumstances. This approach to comparability suggests (among other things) that we consider equivalent two acts of control if they imply changes in patterns of behaviors which are equally important to the controlled individuals. Or, equally probable changes in a given behavior may imply different amounts of power if the affected behaviors differ in importance to $a$.

While this approach to comparability has been developed within the framework of a broad theoretical system, it takes into account some intuitive notions of power suggested by prior research. March (1956), for example, presents data in which correlation between amount of influence attributed by groul members to each other and opinion changes of group members is higher when the opinions relate to a subject of "importance" to members, than when the subject is relatively unimportant. Group members high in attributed influence can be expected characteristically to exercise influence over relatively important issues. This is one reason why they are seen to have high influence. Tannenbaum and Kahn have noted that union members, in evaluating the amount of control which groups within the union exercise over various union activities, appear to evaluate this influence in terms of the "importance" to the members of the controlled activities (Tannenbaum, 1959; Tannenbaum \& Kahn, 1958). French, Israel, and Ås (1960) acknowledge the relevance of "importance" in considering the effects on work production and job satisfaction of increased worker participation in decision-making. Predicted effects will occur, according to these authors, to the extent that the decisions being made are "important" to the workers. One might say that it is only under these circumstances that the workers actually exercise some power. Morse and Reimer (1956) imply a similar process in relation to an experiment dealing with changed loci of control within a clerical organization. ${ }^{2}$ And this is essentially Dubin's position when he suggests defining power in terms of the "importance" of the consequences of the act controlled (1957).

It is interesting to note that, despite its central importance, the problem of power comparability has been virtually ignored by psychologists. Comparability, of course, is as much a problem of theory as of measurement technique. While event-structure theory, upon which our proposed solution is based, provides a first-approximation, theoretical solution, a great deal of work remains to make this solution fully operational. Nevertheless the approach which we offer has immediate implications for research as well as for the theory of power. It is our intention in this paper to define power in event-structure terms, to indicate the relevance of this definition for the problem of comparability, and to outline some of its theoretical and empirical implications.

\section{EVENT-STRUCTURE THEORY}

Allport has presented an introduction to his system in several publications (1954, 1955, 1962) and we must assume some familiarity on the part of the reader with these writings. We shall describe here only very briefly the

\footnotetext{
2 The authors do not elaborate this point in their only published report of their experiment. The above statement is based on personal communication.
} 
few event-structure concepts which apply directly to our problem.

"A basic postulate of the system is that behavior can be described as a cycle of [ongoings and] events or as a system of such cycles in interrelationship. The contention that the behavioral pattern is a true cycle is based on the fact (or likelihood) that the [ongoings and] events which make it up return to the region in which they started. For example, 'drinking' usually starts with drying in the mouth or throat, and the [ongoings and] events that ensue (involving receptors, neurons, muscle fiber contractions, etc.) bring the cycle of ongoings back to the same region, as the membrane of the mouth and throat is moistened. This feature of return to the starting region is known as 'closure.' The throat-drying represents a 'primary' event (really many [ongoings and] events in a region), which constitutes the initial energic upset of the cycle. The act of drinking involves a displacement or equalization of energy of the primary region. It is hypothesized that all behavioral acts, overt or covert, can be described in terms of such selfclosed cycles or structures.

"Event-structure theory, which is a general conception, provides for the 'group' or collective situation in the same way. It is conceived as a structure of a higher order that is built, on the same principles, from the structures of individuals' behavior. Events [and ongoings] occur between individuals; and depending on probabilities, the events [and ongoings] proceed from energic upset in one part (or individual) around the collective cycle and back to the initial region, in which an intraorganismic equilibrium of the participant is then attained. We have, therefore, a collective structure ... as well as the individual behavior structures [cycles] that compose it" (Tannenbaum \& Allport, 1956, pp. 272-273).

The exercise of power is a type of collective structure in which behavior on the part of the controlled individual provides some of the ongoings and events leading back to the primary event region of the individual exercising control. The controlled behavior, though providing some of the ongoings and events in a cycle leading to closure within the person exercising control, forms, at the same time, ongoings and events of a second cycle leading to closure within the individual being controlled. This illustrates a type of relationship between cycles.

Cycles relate to one another through the process of "interstructurance." One cycle is positively interstructurant with another when it contributes to or supports the operation of the latter. The relationship is said to be negatively interstructurant when one cycle impinges on another so as to limit or prevent the latter's operation. This concept, of central importance in event-structure theory, proves valuable to our analysis of social power. It leads to a consideration of the full implications (i.e., the positive and negative interstructurances) of the collective cycle involving the influence attempt on the other cycles which form the "behavioral repertory" of the individual being controlled.

Each behavior cycle or structure consists of three elements: a primary or initial state of energic upset; a series of ongoings and events, which usually involve the organism and its environment; and closure, which represents the return of the initial energic upset to a state of equilibrium. Amount of closure is defined quantitatively as:

$$
C=e_{1}-e_{2}
$$

where $e_{1}$ is the amount of the primary event's energy before closure and $e_{2}$ the amount of its energy remaining after the cycle's operation (i.e., after some closure). All behavior involves this reduction of the initial primary event energies. If closure is complete, that is, all the initial energies are completely reduced, $e_{2}=0$ and $C=e_{1}$. The concept of motivation (needs, drives, motives, etc.) is concerned essentially with these energies (Allport, 1955 , p. 654).

It should be noted that the assumption (especially important to our proposed solution) of an energic basis for human motivation and behavior is not unique to eventstructure theory. Murphy, for example, posits "energy systems" as the basis of human motivation and argues that ... "all activity is traceable to tension, that tension is 'need' for acting, and that tension, need, and motive are 
one and the same. The term 'tension' is used as in physics" (1947, p. 89, italics added). The position of Murphy and that of Allport is not to be seen as offering the concept of energy (or of tension) as a kind of psychological analogy to the physical concept. Energy is to be understnod in its literal, physical sense. "The concept of tension developed here necessarily entails the notion that there exist certain objects in the universe which will raise or lower the tension when contact with them is macle. ... The tense condition of tissues is a mechanical, electrical, or chemical tension. ... The tension is reduced (kinetic energy taking its place) when contacts are made with certain other objects. ..." (Murphy, 1947, p. 165, italics added. Miller [1955] presents a relateıl view.)

We prefer this physicalistic approach to motivation partly because it provides an underlying conceptual basis for comparability among "need" intensities within and between individuals. Many readers who hold a conception of motivation involving the analogy of "psychological energies" or "tensions" may nevertheless find useful the approach to power which is developed below by substituting the appropriate "psychological" constructs into our formulation.

While adequate, "physical" measures of motivational energies are not now available, indirect measures (including phenomenological and objective) are possible under certain circumstances. Furthermore, the reader will see below how it may be possible to manipulate $e$ values experimentally. While these operations and manipulations may lack precision, they can serve as reasonable approximations under controlled conditions.

The event-structure analysis of behavior is thus premised on the existence of a large but finite number of primary event energies within the organism which are available for interstruct uring with other ongoings and events into behavior cycles. Whether or not such an interstructuring occurs depends on the availability (which can be conceived as probabilities) of in series, organismic, and environmental means.

We have proposed that the "mechanism" of choice may provide a clue to the solution of the problem of comparability. Let us therefore take choice behavior as an example. The alternative choices represent behaviors which, as "in series ongoings and events," provide the means through which initial energic upsets may return to a state of equilibrium. Allport's notion of "means value" or of the "degree of positive relevance" is useful here. The degree of positive relevance of a behavior to a need-state is "the proportion of the maximal energic disturbance $\left[e_{1}\right]$ produced by the need-state that ... [the behavior] will bring to equilibrium. The more fully the ... [behavior] will satisfy the need, that is, the greater the fraction of the energic upset it will 'equilibrize,' the greater will be its ... [relevance]" (Allport, 1955, p. 353).

If we postulate that behavior tends toward the maximization of "closure" we can predict an individual's choice by knowing the intensities of his various primary event energies and the degree to which each of the potential behaviors will provide means for the reduction of these energies. We thus have a set of primary event energies with $e_{1}$ values, $e_{1 \alpha}, e_{1 \beta}$, $\ldots e_{1 \eta}$, and the "relevances" of a given behavior, represented, let us say, by choice $I$, to each. We can designate such relevances of choice $I$ to each of the primary event energies by the symbols: $R_{I a}, R_{I \beta}, \ldots R_{I \eta}$. If we call the amount of energy reduction likely to result from choice I $\Sigma\left(e_{1}-e_{2}\right)_{t}$, (Tannenbaum, 1954; Tannenbaum \& Allport, 1956) we can state that:

$$
\begin{array}{r}
\Sigma\left(e_{1}-e_{2}\right)_{I}=e_{1 a} R_{I a}+e_{1 \beta} R_{I \beta}+ \\
\ldots e_{1 \eta} R_{I \eta}=\sum_{a}^{\eta} e_{1} R_{I} .
\end{array}
$$

In the case of choice II, the statement becomes:

$$
\begin{array}{r}
\Sigma\left(e_{1}-e_{2}\right)_{I I}=e_{1 a} R_{I I a}+e_{1 \beta} R_{I I \beta}+ \\
\ldots e_{1 \eta} R_{I I \eta}=\sum_{a}^{\eta} e_{1} R_{I I} .
\end{array}
$$

Assuming, for the sake of illustration, that only two alternatives (choice I and choice II) are possible, the probability of choice I can be stated thusly:

$$
P_{I}=\frac{\Sigma e_{1} R_{I}}{\Sigma e_{1} R_{I}+\Sigma e_{1} R_{I I}}
$$


and $P_{I l}$ becomes:

$$
P_{I I}=\frac{\Sigma e_{1} R_{I I}}{\Sigma e_{1} R_{I}+\Sigma e_{1} R_{I I}} .
$$

The choices might represent widely disparate behaviors, based ostensibly on qualitatively different "reasons" (e.g., the chosen job is better, pays more, is more interesting, etc.). The reasons, however, each have "weight" reflecting probable effects of the choice behaviors on the primary event energies. These energies lie at the basis of all behavior and all behavior in turn has a probable effect on these energies. These energies can be seen as a kind of "common denominator," one kind of standard against which all behaviors, from the most rudimentary and simple to the most evolved and intricate, can be evaluated and compared.

It is important to note that a person need not physically or overtly test each alternative before making his choice. The choice may be arrived at through covert "mental" processes, an "analysis" involving what Allport calls meaning cycles. "We can regard meaning both as an energically reduced state of structure and also as a kinematic modification of some orginally full and overt act, such, for example, as the act of 'handling' or otherwise 'reacting' to an object. The meanings of objects originally come, in other words, from actual dealings with the environment. In this modification of the act-structure, the event-cycle or system concerned has been abridged so that its operation is now largely 'covert' . . . it is still, however, like every other instance of behavior, a self-closed structure. It is a 'meaning cycle' or a cyclical system; and, though some events of contact with the environment are lost from its format, the meaning-cycle still follows, in an abridged way, the pattern of the full overt act which was its genetic basis. In other words, a meaning is simply an energically diminished and kinematically abridged behavioral act (act-structure)" (Allport, 1955, p. 655). The $P_{I}$ and $P_{I I}$ values of formulas (4) and (5) represent, in effect, the results of a "covert test" involving the operation of meaning cycles and their interstructurances under our assumption of a perfect congruence with the overt behavior cycles and the inter- structurances that would occur in an overt test of the alternatives. Likewise, $\Sigma\left(e_{1}-e_{2}\right)_{1}$ and $\Sigma\left(e_{1}-e_{2}\right)_{I I}$ of formulas (2) and (3) represent the primary event energies that would be reduced in an overt test of the respective alternatives. $P_{I}$ and $P_{I I}$ are therefore approximations made on the assumption that a high degree of correspondence between the covert and the overt processes does generally exist. In considering this assumption, however, one should be careful to distinguish between the behavior of making the choice itself, and the behavioral consequences of that behavior, i.e., the future consequences of the choice. It is to the former, limited behavior that this paper refers.

\section{THE NOTION OF POWER}

A definition of power (or of any concept) is likely to prove all the more useful if it can be developed within the framework of a general theoretical system. ${ }^{3}$ Few definitions of power so far proposed can be said to meet this desideratum. Lewin (1951) has provided a field theoretical definition and Cartwright (1959b) and French (1956) offer analyses of power within this framework. Adams and Romney (1959) suggest a Skinnerian learning theory definition of authority. Simon also provides a statement of power within a broader model of behavior $(1957$, p. 5 ) and related formal treatments are offered by March (1955) and by Dahl (1957). In presenting an approach to power within the framework of event-structure theory, we accept as a starting point the definition proposed by Dahl. Dahl (1957, p. 205) defines " $A$ 's power over $a$, with respect to the response $x$, by means of [influence attempt] $w$, as $M$, or more fully:

$$
\begin{aligned}
M\left(\frac{A}{a} w, x\right) & =P(a, x / A, w)- \\
& P(a, x / A, \bar{w})=p_{1}-p_{2} "
\end{aligned}
$$

3 Due to limitations of space we shall confine ourselves to an analysis of the dyadic power relationship involving only one influence attempt. The author will be happy to send the interested reader a statement covering the application of this approach to situations involving groups and collectivities as well as pluralities of influence attempts. 
where:

$(A, w)=A$ does $w$, representing an influence attempt over $a$;

$(A, \bar{w})=A$ does not do $w$;

$(a, x)=a$ does $x$, representing accession to the influence attempt;

$(a, \bar{x})=a$ does not do $x$;

$P(a, x / A, w)=p_{1}=$ the probability that $a$ will do $x$ when $A$ does $w$;

$P(u, x / A, \vec{w})=p_{2}=$ the probability that $a$ will do $x$ if $A$ does not do $w$.

$A$ is called the actor and $a$, the respondent.

Dahl's approach is consistent in a general way with those of March (1955) and of Simon (1957), who consider power a special case of "causation." Dahl's definition can be understood as referring to that special subset of "cilusal" relations he chooses to call power. His statement conforms to the usual causation paradigm: $w$ is said to be the cause of $x$ if $x$ occurs when $w$ occurs but will not occur if $w$ does not occur.

The amount of power of an actor over a respondent, then, is the probability that the respondent does what the actor "requests" minus the probability that the respondent would have done it in the absence of the "request." It is interesting to note that this formula provides for negative values, or what Dahl calls "negative power."

In event-structure theory, however, power cannot be divorced from the concepts of structure and of energy, and if we are talking about the power of individual $A$ over $a$, we should consider the primary energies of $a$ which are involved in this relationship. We shall therefore recast Dahl's formula in terms of event-structure theory as follows:

4 "This is not as foolish as it may seem, although one must admit that negative control of this kind is not ordinarily conceived of as power. If, whenever I ask my son to stay home on Saturday morning to mon the lawn, my request has the inevitable effect of inducing him to go swimming, when he would otherwise have stayed home, I do have a curious kind of negative power over him. The Legion of Decency somutimes seems to have this kind of power over moviegoers. Stalin was often said to wield negative power over the actions on appropriations for foreign aid by the American Congress" (Dahl, 1959, p. 205).

$$
\begin{aligned}
& M_{e}\left(\frac{A}{a} w, x\right)=[P(a, x / A, w)- \\
& P(a, x / A, \bar{w})] e_{x}=\left(p_{1}-p_{2}\right) e_{x}
\end{aligned}
$$

where $M_{\theta}$ is the event-structure adaptation of the Dahl concept of power, and $e_{x}$ is defined as the primary event energies within $a$ which receive closure through behavior $x$.

A further consideration of $e_{x}$ is necessary at. this point. In thinking of behavior (e.g., eating) in relation to a need (e.g., hunger) it is not uncommon to take the need as a "given" and consider the extent to which the behavior is instrumental in satisfying this need. We thus talk about the degree of satiation or deprivation of a need. We might reverse this process, however, taking the behavior as a given, and consider only those motivational energies which are reduced by that behavior. We are talking here, in a sense, about "parts" of needs. This, in effect, defines the quantity of motivational energies, $e$, as $e_{1}-e_{2}$. $e$ is defined, then, as that part of $e_{1}$ which receives closure through the behavior in question. It is equal to $e_{1} R$, where $R$ is the "relevance" of the behavior to the need structure. $e$ defined in this way proves convenient for our purposes and we shall employ this definition throughout the remainder of this discussion.

Formula (7) implies, then, that the power of $A$ over $a$ with respect to act structure $x$ is greater when the primary energies receiving closure through structure $x$ are large than when they are small. In common parlance, an individual's power over another is likely to be greater when the act controlled is important to the latter than when it is unimportant. Several further distinctions are apparent between formula (7) and Dahl's original conception. Power no longer varies between the limits \pm 1 , but is bounded rather by $\pm e_{x}$. Formula (7) argues that the maximum possible power which an actor might exercise over each of several respondents in relation to a given act $x$ is not a fixed and constant value, but may vary from one respondent to another. Or, the holding constant of probabilities $p_{1}$ and $p_{2}$ of identical (or comparable) behaviors on the part of different respondents may nevertheless imply different degrees of power 
of an actor over these respondents. Furthermore, relatively low values of $p_{1}$ do not necessarily mean relatively low power. Low predictability of accession $\left(p_{1}\right)$ of a behavior very important to the respondent may imply greater actor power than high predictability of an "insignificant" behavior.

We see here the first step in the solution to the problem of power comparability: the primary energies serve as the "common denominator" for equating or comparing the control by $A$ over qualitatively different acts of $a$. But this is only part of the picture. A definition of power which conforms fully to event-structure conceptions would consider the interstructurances (positive and negative) of the cycle, " $A$ influences $a$ " with the other (probable) cycles which make up $a$ 's "repertory of behavorial acts." We would thus consider the total implication of influence attempt $w$ on all of the primary energies of $a$, and on the potential structures through which these energies might receive closure, if $x$ were not to occur. What closures, in other words, does $a$ forego when $x$ occurs? For example, if a student is likely to respond to a professor's request to read an esoteric book, and in the process gives up a movie he is very anxious to see, we would consider this a greater manifestation of professorial power than if the student were likely to read the book partly because he did not have anything better to do anyhow. In neither case is the student likely to have read the book if it were not assigned, i.e., $P(a, x / A \vec{w})=0$; but in giving up the evening movie, the student's behavior is affected more. A greater "segment" of his life is being affected.

Let us use the letters $z_{\alpha}, z_{\beta}, \ldots z_{\eta}$ to represent the various acts which $a$ might perform if $x$ were not to occur, and which $a$ cannot perform if $x$ does occur. Let $e_{a}, e_{\beta}, \ldots e_{\eta}$ represent the corresponding primary energies that would receive closure through these behaviors. The formula now becomes:

$$
\begin{gathered}
M_{e}\left(\frac{A}{a} w, x\right)=\left[p_{1}-p_{2}\right]\left[e_{x}+P\left(a, z_{a} / A, \bar{w}\right) e_{a}+P\left(a, z_{\beta} / A, \bar{w}\right) e_{\beta}+\right. \\
\left.\ldots P\left(a, z_{\eta} / A, \bar{w}\right) e_{\eta}\right] .
\end{gathered}
$$

This formula weights the probabilities $\left(p_{1}-p_{2}\right)$ by the motivational energies involved in the act $x$ as well as the motivational energies that are likely to remain unclosed (i.e., frustrated) as a result of act $x$. All of the motivational energies affected by act $x$, in the sense that act $x$ either affords or prevents their closure, are thus taken into account.

While this seems both an intuitively and theoretically satisfying formulation, the operational problem involved in this definition is formidable. A reasonable approximation, however, may vastly simplify the above formula. Let us say that $e_{c}$ represents the sum of all the individual's primary event energies, considered above, exclusive of $e_{x}$.

$$
e_{c}=e_{a}+e_{\beta}+\ldots e_{\eta}
$$

The approximation follows:

$$
\begin{aligned}
& P\left(a, z_{a} / A, \bar{w}\right) e_{a}+P\left(a, z_{\beta} / A, \bar{w}\right) e_{\beta}+ \\
& \quad \ldots P\left(a, z_{\eta} / A, \bar{w}\right) e_{\eta} \cong e_{c} \Sigma P(a, z / A, \bar{w})
\end{aligned}
$$

But:

$$
\Sigma P(a, z / A, \bar{w})=1-P(a, x / A, \bar{w})
$$

$$
\begin{aligned}
\therefore M_{e}\left(\frac{A}{a} w, x\right)=[P(a, x / A, w)- & P(a, x / A, \bar{w})]\left\{e_{x}+e_{c}[1-\right. \\
& P(a, x / A, \bar{w})]\}=\left(p_{1}-p_{2}\right)\left[e_{x}+e_{r}\left(1-p_{2}\right)\right]
\end{aligned}
$$

where $p_{1}$ and $p_{2}$ are defined in formula (6).

This approximation may be justified as an operational expedient since $e_{c}$ is probably easier to measure as a totality than are all of the individual $e^{\prime} s\left(e_{\alpha}, e_{\beta} \ldots e_{\eta}\right)$ of a respondent. The mathematics too are simplified by 
this approximation. However, it should be noted that aside from providing a certain degree of simplicity and convenience, this approximation does not alter in any way the principle of our proposed solution.

We thus broaden the concept of power to include not only what $A$ "makes" $a$ do, but what he "prevents" $a$ from doing. Furthermore, we take into account the level of $a$ 's motivation relative to these various actual and potential structures. By applying this formula, we can make meaningful comparisons between the powers of any $A$ 's over any $a$ 's through any acts ( $w$ 's) of the $A$ 's and with respect to any acts ( $x$ 's) of the $a$ 's. We can thus compare a mother's power in getting her child to wash his ears with a professor's power to get a student to read a homework assignment. The quantities of energy which "motivate" the child and those "motivating" the student provide a basis upon which the behaviors of these persons, and consequently the power exercised over them, can be compared.

\section{POWER AND ENERGY}

A further clarification of the above formulation can be derived from the fact that $p_{1}$ and $p_{2}$ are not independent of $e$. $p_{1}$ and $p_{2}$ represent, essentially, the probabilities of certain choices, and the relationship which follows from the postulates stated in formulas (2) and (4) can be written as follows:

$$
p_{1}=\frac{e_{x}}{e_{x}+e_{c}} .
$$

This states simply that the probability of $a$ 's choosing to perform act $x$ is equal to the ratio of the amount of the primary energies that would be reduced through the choice of that act, to the primary energies that would be reduced through the choice of that or any alternative (and mutually exclusive) acts.

$p_{2}$ would be defined similarly. However, it is necessary to consider first an important aspect of $e_{x}$. Like any primary event energy, $e_{x}$ is likely to have at least several sources or components. One might think specifically of $e_{x}$ having two components; that which is attributable to the interaction between $A$ and $a$, and that which is independent of this interaction. In other words, the relationship between
$A$ and $a$, and the influence attempt by $A$ in particular, have the specific effect of creating a certain energy imbalance within $a$ which would receive closure through act $x$. If we denote the primary energies which receive closure through act $x$ and which are attributable to this interaction as $e_{p}$, we can say that:

$$
e_{x}=e_{(x-p)}+e_{p}
$$

where $e_{(x-p)}$ denotes the energies in $e_{x}$ which receive closure through act $x$ but which are independent of the interaction between $A$ and $a$. $p_{2}$ can now be defined as:

$p_{2}=\frac{e_{(x-p)}}{e_{(x-p)}+e_{c}}=\frac{e_{x}-e_{p}}{e_{x}-e_{p}+e_{c}}$

Substituting into formula (12), $M_{e}$ becomes:

$$
\begin{gathered}
M_{e}=\left[\frac{e_{x}}{e_{x}+e_{c}}-\frac{e_{x}-e_{p}}{e_{x}-e_{p}+e_{c}}\right] \\
{\left[e_{x}+e_{c}\left(1-\frac{e_{x}-e_{p}}{e_{x}-e_{p}+e_{c}}\right)\right] .}
\end{gathered}
$$

Defining power in terms of energy values has some interesting implications. First, it makes apparent an intriguing resemblance between social power and the physical concept of power, the rate of doing work (or the rate of energy expenditure). We can derive for example, a value $\frac{M_{\theta}}{\text { time }}$ for any period of time. ${ }^{5}$ This represents a weighted rate of energy $\left(e_{x}\right)$ expenditure. The work being done occurs within the person being controlled. We thus ascribe a degree of social power to an individual according to a type of physical power which occurs within another. The social power of an actor over a respondent represents a type of physical power within the respondent involving the dissipation of motivational energies, which are alifected in a specified way by the actor. Social power can be understood to be a function of the quantity of the re-

5 The reader is asked to envision a graph in which time is the abscissa and $M_{e}$ values corresponding to behaviors in compliance with influence attempts are plotted along the ordinate. $\frac{M_{e}}{\text { time }}$ represents, in effect, the average $M_{e}$ value for a given unit of time. 
spondent's motivational energies which are at the disposal of the actor.

A second implication comes from exploring the behavior of our formula under varying conditions. For example, we can see that $\boldsymbol{M}_{e}$ is zero when $e_{p}$ is zero. This means simply that an actor has no power over a respondent unless an increment of motivational energy to perform the behavior in question occurs as a result of the actor's behavior. This seems intuitively reasonable. $M_{e}$ is also zero when $e_{c}$ is zero. This means that unless the respondent has some motivation to do something other than the behavior in question, "inducing" him to perform this behavior does not represent power. This reflects Dahl's notion that the actor's power is zero if the respondent would have performed the requested behavior in the absence of an influence attempt. We can also see that $M_{e}$ is zero when $e_{x}$ is zero. This follows from the fact that $e_{p}$ must be zero when $e_{x}$ is zero since $e_{x}=e_{(x-p)}+e_{p}$, and negative energy values do not occur. This states, in effect, that if there is no motivation to perform the behavior $x$, it will not occur, and power, of course, must be zero under these circumstances.

$e_{p}$ is an especially interesting element in our formula since it represents that increment of the respondent's motivational energy to perform the requested behavior, which is attributable to the interaction between actor and

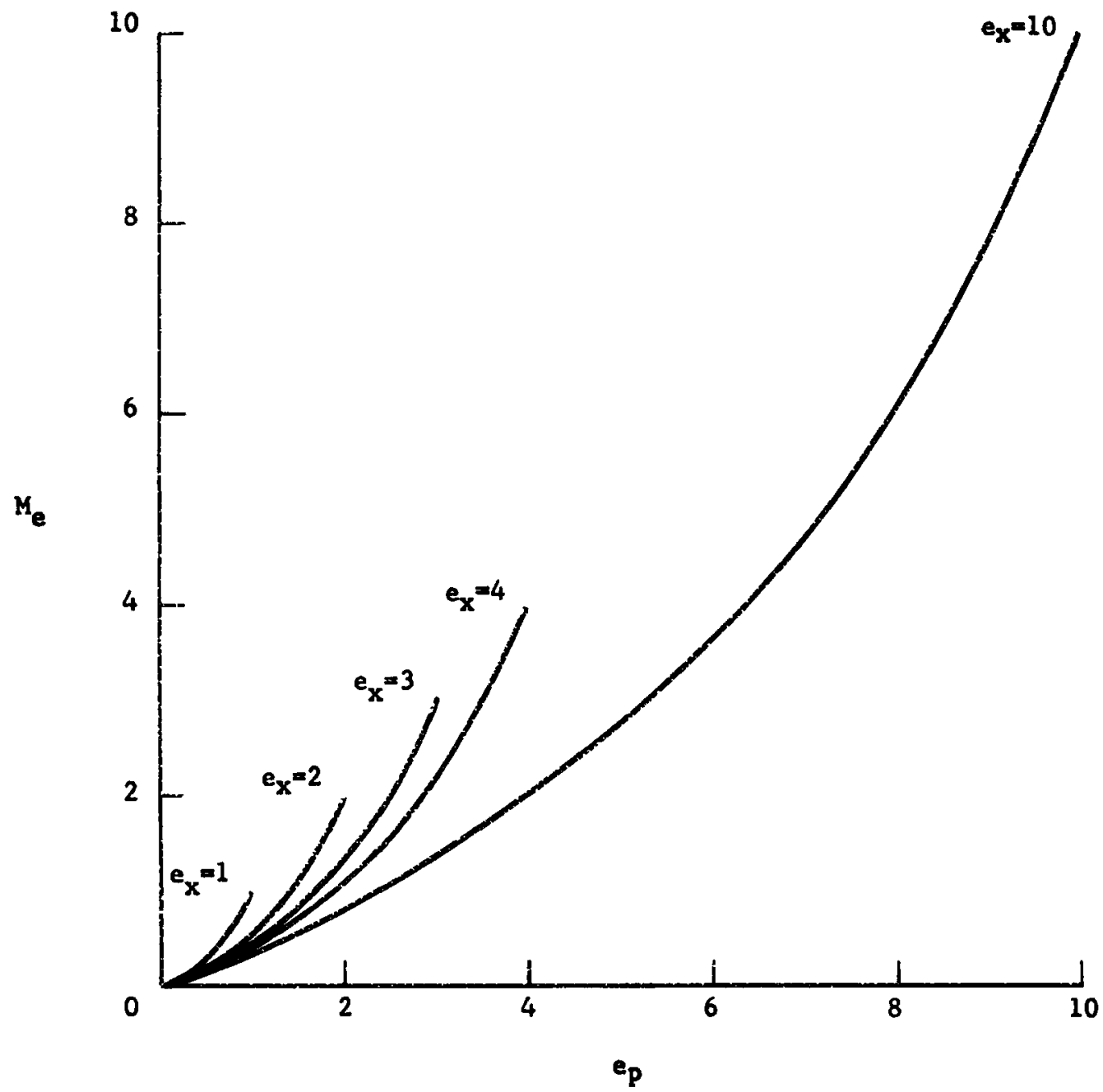

Fic. 1. The Relationship Between $e_{p}$ and $M_{e}$ for Different Values of $e_{x}$ under the Condition $e_{x}=e_{\theta}$. 
responilent. It is therefore of interest to see how variations in it may affect $M_{e}$. Figure 1 shows several parameters describing the relationship between $e_{p}$ and $M_{e}$ under the condition that $e_{x}=e_{c}$. It is clear, first of all, that for each parameter $M_{e}$ tends to increase with $e_{p}$. This would seem to satisfy our intuitive understanding of power. Furthermore, the relationship is not linear. Power increases more rapidly with increments of $e_{p}$ when $e_{p}$ is large. This would be consistent with the hypothesis that "inducing" small values of $e_{p}$ is relatively easy, but larger and larger increments of $e_{p}$ become progressively more difficult to "induce." Power therefore might reasonably be expected to increase at a faster rate with unit increments of $e_{p}$ when $e_{p}$ is large.

It is also interesting to note that power cannot be great when $e_{x}$ is small since $e_{p}$ can never be greater than $e_{x}$. Finally, note that increasing $e_{x}$ while holding $e_{p}$ constant has the effect of decreasing power. This is explained by the accompanying increase in $e_{(x-p)}$ and the consequent increase in $p_{2}$. Conversely, increasing $e_{x}$ while holding $M_{e}$ constant must be accompanied by an increase in $e_{p}$.

\section{NEGATIVE POWER}

It will be noted that since $e$ can never be negative, $\frac{e_{x}-e_{p}}{e_{x}-e_{p}+e_{c}}$ (i.e., $\left.p_{2}\right)$ can never be greater than $\frac{e_{x}}{e_{x}+e_{e}}$ (i.e., $\left.p_{1}\right)$, and "negative power" as defined by Dahl will not occur in formula (16). However, one of the consequences of the interaction between $A$ and $a$ may be the creation of primary event energies which will receive closure through alternative behavior. Let us take Dahl's (1957) example of the son who suddenly "becomes motivated" to go swimming when his father asks him to mow the lawn. The motivational energies involved in the desire to go swimming, attributable to the father's request, now become a component of $e_{r}$. If we call this component $e_{n p}$ we can say that:

$$
e_{c}=e_{(c-n p)}+e_{n p}
$$

Now we can see the possibility of negative power since:

$$
p_{2}=\frac{e_{w}-e_{p}}{e_{x}-e_{p}+\left(e_{e}-e_{n p}\right)}
$$

and:

$$
\begin{aligned}
\boldsymbol{M}_{e}=\left[\frac{e_{x}}{e_{x}+e_{e}}-\right. & \left.\frac{e_{x}-e_{p}}{e_{x}-e_{p}+e_{x}-e_{u p}}\right] \\
& {\left[e_{x}+e_{c}\left(1-\frac{e_{x}-e_{p}}{e_{x}-e_{p}+e_{e}-e_{n p}}\right)\right] }
\end{aligned}
$$

It is clear that $p_{2}$ can be greater than $p_{1}$ in this formula, and $M_{e}$ therefore negative. For example, $M_{e}$ will be negative if $e_{p}=0$ and $e_{n p}>0$.

The negative sign has been associated with the power relationship in quite another sense than that proposed by Dahl. Meyers (1944) talks about "positive" and "negative authority," i.e., situations in which an individual is told (asked, ordered, requested, etc.) to do something versus situations in which an individual is told not to do something. (See also Johnson. 1939; Langfield, 1913.) This distinction can be handled by noting that "positive authority" ordinarily implies a single $x$ and a number of $z$ 's, while "negative authority" implies a single $z$ and a number of $x$ 's. If an individual is told to do something (a single $x$ ), this ordinarily precludes a number of alternatives ( $z$ 's) which he might otherwise perform. However, if an individual is told not to do something (a single $z$ ), a number of alternatives ( $x$ 's) may remain open to him and consistent with the influence attempt. ${ }^{6}$ If, then,

6 "Positive" and "negative influence attempts vary in their level of generality. They might refer to a specific act at a definite "point" in time, or they might apply to a class of acts during a broad timespan. The Church's interdiction against birth control or the admonition, "Love thy neighbor" illustrate in- 
the definition of $x$ is generalized to include any act in conformance with an influence attempt (rather than the single act implicit in a "positive" influence attempt), formula (19) can be generalized to handle both "negative" and "positive authority" in the Meyers sense, by the appropriate addition of summation signs: aversive stimulation and specifying its own reinforcement, is reinforced by B" (1959, p. 234). Their analysis of the "authority sequence," starting with "deprivation" or "aversive stimulation" within the actor and terminating with "reinforcement," bears an analogous relationship to the event-structure

$$
\begin{aligned}
M_{r}=\left[\frac{\Sigma e_{x}}{\Sigma e_{x}+\Sigma c_{z}}-\frac{\Sigma e_{x}-e_{p}}{\Sigma e_{x}-e_{p}+\Sigma e_{z}-e_{n p}}\right] \\
{\left[\Sigma e_{x}+\Sigma e_{z}\left(1-\frac{\Sigma e_{x}-e_{p}}{\Sigma e_{x}-e_{p}+\Sigma e_{z}-e_{n p}}\right)\right] }
\end{aligned}
$$

where there will be as many $e_{x}$ 's and $e_{z}$ 's as there are $x$ 's and $z$ 's respectively. However, only one $e_{p}$ and one $e_{n p}$ exist for a single influence attempt. It will be remembered from formula (9) that $\Sigma e_{z}=e_{c}$.

Formula (20), a more general statement than (19), can be seen to reduce to (19) under conditions of "positive authority." "Positive authority" is, in our event-structure view, a limiting case of "negative." We shall elaborate the meaning of formula $(20)$ in a discussion below of some of its specific research implications.

\section{COMPARISON WITH OTHER APPROACHES}

We have already mentioned briefly the relationship of our proposed definition of power to those of Dahl (1957), March (1955), and Simon (1957). Adams and Romney offer quite a different approach. They define "authority" as follows: "Person A has authority over Person $B$ in a given situation, when a response of $A$, under the control of deprivation or

fluence attempts of a relatively general kind. "Johnny, please bring me the newspaper" or "Johnny, stop teasing your sister" are more specific. It is important to recognize the underlying generalityspecificity continuum on which these influence attempts fall; they differ, insofar as our event-structure formulation is concerned, in degree rather than in kind. While the present conceptualization handles each equally well, it points up the need of making explicit who the actor is (e.g., the "Church," Johnny's father, etc.), precisely what is meant by the positive or negative influence attempt, and when and to whom it applies. "cycle," starting with the "primary node event" and terminating with "closure." While Adams and Romney emphasize in their definition reinforcement of the actor's behavior, we have chosen to emphasize "closures" within the respondent. This is not an inevitable consequence of the event-structure approach, however, and useful analyses of power within an event-structure framework can be made following Adams and Romney's lead. It is important to note, however, that while we define power quantitatively in terms of respondent behavior and closures, we are not disinterested in those aspects of actor motivation and behavior which may affect or be affected by respondent closures. On the contrary, this is the core of the study of power relations, and we would conceive of these variables either as "independent," "intervening," or "dependent" in relation to the power variable as we define it.

\section{The Lewinian approach}

Elements of our proposed definition serve functions analogous to those of certain constructs employed in field theory definitions of power. $e_{p}$ is similar in function to "induced force." $e_{(x-p)}$ brings to mind a combination of "own," "impersonal," and "induced" forces "toward" the respondent "goal" $x$. However, the "induced forces" in this case are attributable to persons other than the actor, $A$. $\left(e_{z-n p}\right)$ might represent the same combination of forces "away" from the goal $x$. The Lewinian concept of "motive base," important to the field theoretical analysis of social power, 
can be understood in terms of $e$ values, represented partly in our formula by $e_{x}$ and especially $e_{p}$. For example, certain of French and Raven's "bases of power" (1959) can be seen to represent "needs" (e.g., to avoid punishment, to be rewarded, to please $A$, etc.) which as energic disequilibria comprise $e_{p}$. French. Israel, and Ås (1959) make an important distinction between "resistance" and "opposition." We would regard $e_{n p}$ as the basis of "resistance," while "opposition" would be a function of $e_{(z-n p)}$ and $e_{(x-p)}$. The acts $z_{a}$, $z_{\beta} \ldots z_{\eta}$ together with $x$, are analogous to Cartwright's (1959b) "repertory of potential acts" for $a$, although the use to which this construct is put in the present formulation is quite different from that intended by Cartwright.' Finally, it should be noted that the present formulation can be used in ways analogous to the field theory definitions of "control," "power," or "influence." $M_{e}$ is closest, by analogy, to the field theoretical definition of "control," the production of "... a combined force in $P$ 's [the respondent's] life space ... having a direction which corresponds to that intended by O [the actor]" (Cartwright, 1959b, p. 195). The field theory definitions of "influence" and of "power" can be seen to involve variations of $M_{e}$. For example, "influence" as defined by French and Raven is a function of only $e_{p}$ and $e_{n p}$, i.e., "the influence of $O$ [the actor] does not include $P$ 's [the respondent's] own forces nor the forces induced by other social agents" (1959, 1. 151). "Power" as defined by Cartwright is concerned with a hypothetical potential, or maximum value of "influence" (Cartwright, 1959b).

Certain interesting departures from the Lewinian approach implicit in our proposed formulation can be illustrated by a brief analysis of the special case when $p_{2}=.5$, i.e., $P(a, x / A, \bar{w})=P(a, z / A, \bar{w})$. For the sake of simplicity, we shall assume that $e_{n p}=0$ and that we are working under conditions of Meyers' "positive authority." This means that:

$$
-\frac{e_{x}-e_{p}}{e_{c}-e_{p}+e_{c}}=\frac{e_{c}}{e_{\infty}-e_{p}+e_{c}}
$$

7 Our $u$ ie of this analytic device is similar to the use which March and Simon make of set theory in their analy ses of power (March, 1955; Simon, 1957). and:

$$
e_{x}-e_{p}=e_{r}
$$

Let us impose the condition that $e_{r}>0$. The Lewinian analysis of this circumstance would suggest that the force toward and the force away from the respondent's goal, $x$, before the actor enters the former's field, are equal. The field theory approach would argue that the effect of a given magnitude of induced force, under these circumstances, is independent of the absolute sizes of the original opposing forces because the resultant force will always be equal to the induced force. For example, if the force in the respondent's field toward goal $x$ is ten units and the force away is ten units, before the actor enters the field, and the latter then adds a force of five units toward $x$, the resultant "force" is five units toward $x$. If the original forces had been 20 , 40 , or any value, the resultant would still be five units.

The present analysis suggests that the power of an actor under these circumstances may vary widely depending upon the magnitudes of $e_{x}, e_{p}$, and $e_{c}$. Holding $p_{1}$ and $p_{2}$ constant (and fixing $p_{2}=.5$ ), power will increase as $e_{(x-p)}$ or $e_{c}$ increase. This says, in effect, that the power of an actor over a respondent is greater if the act likely to be performed, or the other acts which the respondent might perform, are important to the respondent than if they are not.

A hypothetical example may be useful in clarifying this point. Let us say that $I$ am about to go to the movies but am undecided as to which of two films I should see. I am equally interested in both, and the probability that I shall go to one equals the probability that I shall go to the other. I might even toss a coin to decide (i.e., $p_{2}=.5$ ). But a friend suggests that I see movie $Y$ and the probability that I shall follow his advice is high. This represents a certain power, however moderate, which my friend may exercise over my behavior. Now suppose I am about to buy a new house and after careful investigation and consideration I am undecided between two models. Each of the houses is expensive and would involve my life's savingsand I like both equally (i.e., $p_{2}=.5$ ). $\mathrm{My}$ friend advises that I purchase house $Y$, and 
again the probability that I shall follow his advice is high. Surely this represents a relatively high degree of power. $\boldsymbol{M}_{e}$ would certainly be higher under these circumstances since $e_{i r}$ and $e_{r}$ are quite high. It is hard to see, however, how the Lewinian approach would indicate adequately the increased power represented in the house-buying as compared to the movie-going situations.

\section{SOME RESEARCH IMPLICATIONS}

The value of our event-structure formulation rests partly on its usefulness as a conceptual tool, as a means of thinking about and analyzing social situations in which "power" may be said to be present. However, it is possible to manipulate experimentally the various elements of our formula and to test hypotheses deduced from some of the postulates upon which it is based. The definition itself also suggests a number of hypotheses, as we shall see. The following illustrate briefly several possible manipulations.

$e_{p}$ might be manipulated through varying the level of rewards or punishments administered to subjects ( $a$ 's) by the experimental actor $(A)$; through varying the character of the actor, for example, a prestigeful versus a nonprestigeful person; through varying the nature of the influence attempt, for example, "Engage in that activity if you like" versus "The experiment requires that you engage in that activity" (Frank, 1944a). $e_{(z-n p)}$ might be manipulated by techniques of satiationdeprivation. For example, a respondent who is hungry can be assumed to be higher on $e_{z-n p}$ than one who is not hungry (all other things equal), providing the behavior in which he is asked to engage $(x)$ is not eating. If it is eating, we have manipulated $e_{(x-p)}$. $e_{(x-p)}$ and $e_{1 z-n p)}$ can also be manipulated by setting up a variety of tasks or activities for a subject, each of which represents a different degree of "attractiveness" for him. Being asked to engage in an "attractive" activity would imply a relatively high $e_{(x-p)}$ value as opposed to being asked to engage in an "unattractive" activity. Similarly, being asked not to engage in an "attractive" activity implies a relatively high value of $e_{(z-n p)}$.

The experimental manipulation of $e_{n p}$, al- though extremely interesting, is perhaps more problematic since it is concerned with what Dahl refers to as "negative power" (1957) and with what the Lewinians call "resistance" (French, Israel, \& Ass, 1960; French, Morrison, \& Levinger, 1960; Sampson, 1960; Zipf, 1958). ${ }^{8}$ A number of possibilities deserve experimental investigation. We shall mention one briefly. In the Sampson experiment (1960) the experimental actor, $A$, behaved so as to antagonize $a$, provoking hostility and rebelliousness on the latter's part. $a$ was thus "motivated" not to comply. A's observable discomfort at $a$ 's noncompliance would serve, in terms of the Adams and Romney authority paradigm (1959), to "reinforce" $a$ 's response of noncompliance.

A large number of experiments are possible employing some of the above manipulations. In the limited space available, one example can be illustrated in only very rough outline. The reader is asked to assume that the manipulations and measures suggested can be improved through pretesting, and that many details not here discussed would be worked through before the proposed experiments are run. Some of the hypotheses stated below are subject to qualifications which we cannot elaborate in this brief treatment. We intend now only to suggest the general research feasibility of our conception and to indicate several directions in which we are led by it.

\section{An experiment}

We have chosen to illustrate the research implications of our approach by describing one experiment concerning the relative effects of "negative" and "positive" authority (Meyers, 1944). This experiment is designed in

8 Frank's early experiments on the production of resistance are suggestive (Frank, 1944a, 1944b). Formulated partly in Lewinian terms, these experiments include manipulations of $e_{(x-p)}, e_{(z-n p)}, e_{p}$, and $e_{n p}$. However, Frank makes no attempt to distinguish these (or their Lewinian counterparts) systematically, and differences in compliance among subjects which he attributes to "resistance" may in some cases be due to variations in "induced force" (i.e., $\left.e_{p}\right)$. Frank does not make the important distinction, later suggested by French, Israel, and $\AA$ s, between "opposition" (a function of $e_{(x-p)}$ and $e_{(z-n p)}$ ) and "resistance" (a function of $e_{n p}$ ). 
TABLE 1

Experinfental Design to Test the Relatrve Effects of Positrve and Negative Authority Under Varying Combinations of $e_{p}$ AND $e_{n p}$

\begin{tabular}{|c|c|c|c|c|}
\hline \multirow[b]{2}{*}{$e_{n p}$} & \multicolumn{2}{|c|}{$\begin{array}{l}\text { Negative Authority } \\
\qquad e_{p}\end{array}$} & \multicolumn{2}{|c|}{$\begin{array}{l}\text { Positive Au:heri:y } \\
e_{p}\end{array}$} \\
\hline & $\begin{array}{c}\text { High } \\
\text { (prestigeful } A \text { ) }\end{array}$ & $\begin{array}{c}\text { Low } \\
\text { (nonprestigeful } A \text { ) }\end{array}$ & $\begin{array}{c}\text { High } \\
\text { (prestigeful } A \text { ) }\end{array}$ & $\begin{array}{c}\text { Low } \\
\text { (nonprestigeful } A \text { ) }\end{array}$ \\
\hline $\begin{array}{l}\text { Low } \\
\qquad(\text { nonantagonistic } A)\end{array}$ & 1 & 2 & 5 & 7 \\
\hline $\begin{array}{l}\text { High } \\
\quad(\text { anta:yonistic } A)\end{array}$ & 3 & 4 & 6 & 8 \\
\hline
\end{tabular}

part to test the view that some of the effects of both of these "types" of authority can be understood in terms of a single formula.

Set up a very large number of activities in which individual subjects might engage in the laboratory (Chinese puzzles, crossword puzzles, reading poetry, listening to jazz records, listening to classical records, etc.). Ask each subject individually to rate the attractiveness of each of these activities, i.e., how much he would like to engage in each. This measure is designed to reflect $e_{(x-p)}$ or $e_{(z-n p)}$, depending upon subsequent experimental manipulations. On the basis of these ratings of the activities by each subject individually, create groups of activities, each of which includes at least ten activities equal (or close) in attractiveness. These groups of activities may be different for different subjects. Place subjects in the laboratory individually. Two experimental manipulations are involved at the outset:

(1) The number of activities which are available to subjects is varied, starting with conditions in which only two activities are available, then three, then four, etc., up to as many as feasible.

(2) The influence attempt is varied. Under one condition it is positive: "John, play with the blocks." Under the other condition it is negative: "John, don't play with the blocks." Variations in the strength of these orders might iorm the basis of further experimental conditions. It is important to use a moderate "strength" of order (i.e., low $e_{p}$ ) such that some clisobedience will occur. Other things are held constant or randomized.
Hypothesis 1. The likelihood of obedience increases under conditions of negative infuence attempt and decreases under conditions of positive influence attempt as the number of available activities increases.

This hypothesis is deduced from the postulate stated in formula (13) and generalized in formula (20) to account for negative authority. Our formula implies families of curves, several parameters of which are shown in Figure 2. The reader will note that Hypothesis 1 follows from any one of the parameters drawn in this figure. Each of these curves is plotted on the assumption of certain relative values of $e_{(x-p)}, e_{(z-n p)}, e_{p}$, and $e_{n p}{ }^{9}$ Figure

9 For the reader who is interested in seeing for himself the effects of varying combinations of values for these variables, the appropriate formula is

$p_{1}=\frac{\Sigma e_{x}}{\Sigma e_{x}+\Sigma e_{z}}=\frac{\Sigma e_{x-p}+e_{p}}{\Sigma e_{x-p}+e_{p}+\Sigma e_{z-n p}+e_{n p}}$. Note that since all activities are equally attractive, we can treat each $e_{x-p}$ and $e_{z-n p}$ value as being equal to a constant, $C$. Under circumstances of the positive influence attempt, $\Sigma e_{x-p}=C$ and $\Sigma e_{z-n p}=$ $(N-1) C$, where $N$ is the number of activities available during a given experimental condition. Under circumstances of the negative influence attempt, $\Sigma e_{z-n p}$ $=C$, and $\Sigma e_{x-p}=(N-1) C$. The various parameters of Figure 2 are drawn by assigning values to $e_{p}$ and $e_{n p}$ relative to $C$. We assume further that $e_{p}$ and $e_{n p}$ are functions of the actor, the respondent, and the influence attempt (whether positive or negative). As long as these three factors are held constant, $e_{p}$ and $e_{n p}$ remain constant, although not necessarily equal to each other. In varying the number of avai'able alternatives while holding the actcr, respondent, and influence attempt constant, we are following one or another of the parameters drawn in Figure 2. 
2 suggests why differences ordinarily found between the effects of positive and negative influence attempts may not always be in the same direction or why the differences may wash out under certain circumstances.

If we manipulate $e_{p}$ (e.g., a highly prestigeful $A$ versus a nonprestigeful $A$ ) and $e_{n p}$ (e.g., an "antagonistic" $A$ versus a "nonantagonistic" $A$ ), the design represented in Table 1 is possible. A number of hypotheses can be tested through this design. We can condense and simplify their statement as follows:

Hypothesis 2. The obedience curves obtained under each of the eight numbered experimental conditions defined in Table 1 will approximate respectively the numbered curves of Figure 2.

We cannot take the space to elaborate the relevance of respondent personality as a conditioning variable in relation to the above hypotheses. We should mention, however, that it is possible to deduce specific hypotheses which take into account aspects of respondent personality and we would want to modify the above hypotheses (in predictable ways) depending upon aspects of subject personality.

A number of additional hypotheses can be stated relating power to several of the dependent variables of the Meyers experiment (1944): emotional behavior, constructive use

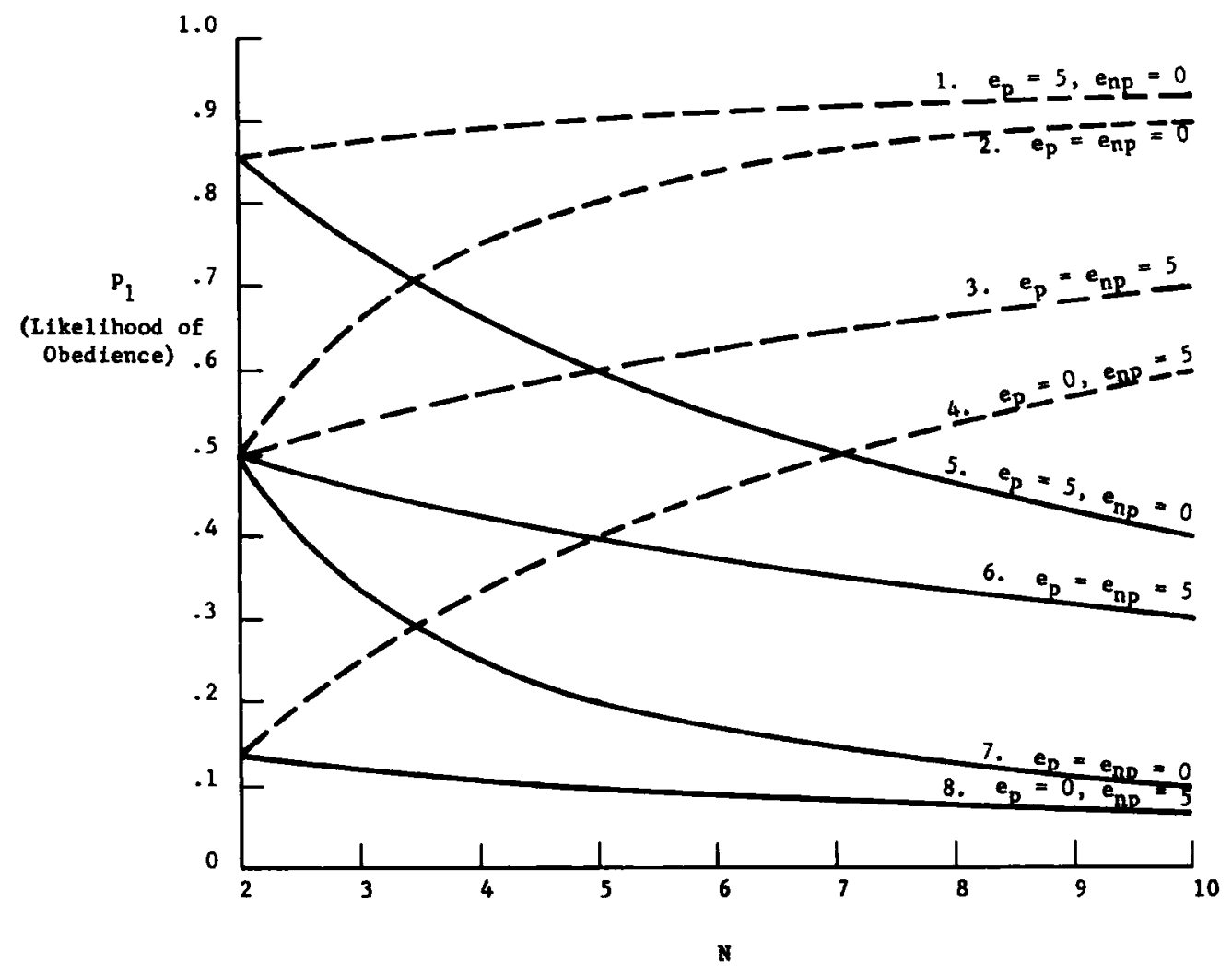

(Number of Avallable Activities)

Frg. 2. Likelihood of Obedience as a Function of Number of Available Activities under Conditions of Negative and Positive Authority for Different Combinations of $e_{p}$ and $e_{n p}$. 
of materials, productivity, and feelings of frustration; as well as respondent's attribution of power to the actor and his feelings of freedom in and attitudes toward the experiment. For example:

Hypothesis 3. Under conditions of either the positive or negative influence attempt, respondents whose curves (Figure 2) approach the straight line, $p_{1}=.5$, will express greater ambivalence toward the experimental actor than those whose curves diverge from the line $p_{1}=.5$. The latter will either like, dislike, or be indifferent to the actor.

This hypothesis is based on the deduction that the curve $p_{1}=.5$ will be approached under conditions of relatively high values of botk $e_{p}$ and $e_{n p}$

The following hypothesis illustrates how our definition of power as stated in formula (20) may provide the basis for a number of predictions.

Hypothesis 4. The above dependent variables (power attributed to the actor, satisfaction with experiment, feelings of freedom, etc.) are a function (direct in some cases, inverse in others) of the power exercised over the subject (i.c., $M_{\mathrm{e}}$ ).

We cannot, in the limited space available, describe each of the relationships implicit in Hypotbesis 4 nor the specific bases upon which they would be formulated. In general, these relationships can be stated in terms of predicted different slopes of $M_{*}$ curves under the conditions of "positive" and "negative" influence attempts. The following hypothesis is an illustration:

Hypothesis 4a. The amount of power attributed to the experimental actor by respondents will increase with $N$ (the number of available activities) under conditions of "positive authority" and decrease with $N$ under conditions of "negative authority."

"Attributed power" is a variable of considerable importance in the study of power (March. 1955). This hypothesis says that intuitive judgments of power correspond in general to the function $M_{e}$. This is not inconsistent with some available evidence. $M_{\epsilon}$ weights the probabilities of behavior $\left(p_{1}-\right.$ $p_{2}$ ) by the motivational energies involved directly in the act as well as in other acts precluded by the one in question. This, in effect, weights the probabilities by what might reasonably be called the importance or significance to the respondent of his performing the act in question. We have already discussed the studies by March (1956) and by Tannenbaum and Kahn (Tannenbaum, 1959; Tannenbaum and Kahn, 1958) which provide some indication of the relevance to attributed power of the importance of the issues controlled. A study by Levinger (1959) provides complementary evidence regarding the probability aspect of our formula. Levinger's subjects whose influence attempts have a high probability of acceptance $\left(p_{1}\right)$ clearly attribute greater relative power to themselves than do subjects whose influence attempts have a low probability of acceptance. We propose that our event-structure formula, employed as a basis for predicting attributed power, brings together the kinds of findings illustrated by Levinger, March, and Tannenbaum and Kahn.

\section{CONCLUSIONS}

The concept of power which we offer is a matter of definition and one may, of course, define a term as he pleases. The present definition, we believe, has the following advantages:

1. It is consistent with the framework of a very general theoretical system.

2. It corresponds in important respects with certain generally held intuitive notions of power.

3. It is consistent in some essential respects with other current definitions, although it differs clearly from the latter in crucial aspects.

4. It can be useful in experimental research as well as in the analysis of certain empirical events, despite some of its operational difficulties.

5. It offers a theoretical, first approximation solution to the problem of power comparability, a vital problem in the study of power which has been virtually ignored in the psychological literature.

According to Cartwright (1959a), psycholo- 
gists have been "soft" on power. Defining power, as we propose, in terms of motivation, places the study of power within the mainstream of psychological theory and research. We would thus hope to see the study of social power advance, and more satisfactory operational solutions to the problem of comparability develop, with the general progress of psychology in the measurement and study of motivation.

\section{REFERENCES}

Adams, J. S., \& Romney, A. K. A functional analysis of authority. Psychol. Rev., 1959, 66, 234-251.

Allport, F. H. The structuring of events: outline of a general theory with applications to psychology. Psychol. Rev., 1954, 61, 281-303.

Allport, F. H. Theories of perception and the concept of structure. New York: Wiley, 1955.

Allport, F. H. A structuronomic concept of behavior: individual and collective. I. Structural theory and the master problem of social psychology. J. abn. soc. Psychol., 1962, 64, 3-30.

Cartwright, D. Power: a neglected variable in social psychology. In D. Cartwright (Ed.), Studies in social power. Ann Arbor: Institute for Social Research, 1959, pp. 1-14. (a)

Cartwright, D. A field theoretical conception of power. In D. Cartwright (Ed.), Studies in social power. Ann Arbor: Institute for Social Research, 1959, pp. 183-220. (b)

Dahl, R. A. The concept of power. Behav. Sci., 1957, 2, 201-215.

Dubin, R. Power and union-management relations. Admin. Sci. Quart., 1957, 2, 1, 60-81.

Frank, J. D. Experimental studies of personal pressure and resistance. I. Experimental production of resistance. $J$, gen. Psychol., 1944, 30, 23-41. (a)

Frank, J. D. Experimental studies of personal pressure and resistance. III. Qualitative analysis of resistant behavior. J. gen. Psychol., 1944, 30, 57-64. (b)

French, J. R. P., Jr. A formal theory of social power. Psycinol. Rev., 1956, 63, 181-194.

French, J. R. P., Jr., Israel, J., \& Ås, Dagfinn. An experiment in participation in a Norwegian factory. Hum. Relat., 1960, 13, 1, 3-19.

French, J. R. P., Jr., Morrison, H. W., \& Levinger, G. Coercive power and forces affecting conformity. J. abn. soc. Psychol., 1960, 61, 93-101.

French, J. R. P., Jr., \& Raven, B. The bases of social power. In D. Cartwright (Ed.), Studies in social power. Ann Arbor: Institute for Social Research, 1959, pp. 150-167.
Johnson, M. W. Verbal influences on children's behavior. Ann Arbor: Univ. of Michigan Press, 1939.

Langfeld, H. S. Voluntary movement under positive and negative instruction. Psychol. Rev., 1913, 20, 459-478.

Lewin, Kurt. Field theory in social science. (D. Cartwright, Ed.). New York: Harper, 1951.

March, J. G. An introduction to the theory and measurement of influence. Amer. pol. sci. Rev., $1955,49,431-451$.

March, J. G. Influence measurement in experimental and semi-experimental groups. Sociometry, $1956,19,260-271$.

Meyers, C. E. The effect of conflicting authority on the child. In Lewin, K., et al., Authority and frustration: studies in topological and vector psychology III. Ames, Iowa: Iowa State Univ. Press, 1944, pp. 33-98.

Miller, J. G. Toward a general theory for the behavioral sciences. Amer. Psychol., 1955, 10, 513-531.

Morse, N. C., \& Reimer, E. The experimental change of a major organizational variable. J. abn. soc. Psychol., 1956, 52, 120-129.

Murphy, G. Personality, a biosocial approach to origins and structure. New York: Harper, 1947.

Sampson, E. E. An experiment on active and passive resistance to social power. Unpublished doctoral dissertation, University of Michigan, 1960.

Simon, H. A. Models of man. New York: Wiley, 1957.

Tannenbaum, A. S. Mechanisms of control in local trade unions. In J. Barbash (Ed.), Unions and union leadership. New York: Harper, 1959, 109-118.

Tannenbaum, A. S. The relationship between personality and group structure: as seen through attitudinal reactions to differently controlled group structures in an industrial organization. Unpublished doctoral dissertation, Syracuse University, 1954.

Tannenbaum, A. S., \& Allport, F. H. Personality structure and group structure: an interpretative study of their relationship through an event-structure hypothesis. $J$, $a b n$. soc. Psychol., 1956, 53, 272-280.

Tannenbaum, A. S., \& Kahn, R. L. Participation in union locals. Evanston: Row, Peterson, 1958.

Zipf, S. An experimental study of resistance to influence. Unpublished doctoral dissertation, University of Michigan, 1958.

(Manuscript received June 20, 1961) 